JOURNAL OF THEORETICAL

AND APPLIED MECHANICS

54, 2, pp. 353-368, Warsaw 2016

DOI: $10.15632 /$ jtam-pl.54.2.353

\title{
OPTIMIZED RE-ENTRY INPUT SHAPERS
}

\author{
Petr Beneš, Michael Valášek \\ Czech Technical University in Prague, Faculty of Mechanical Engineering Dept. of Mechanics, \\ Biomechanics and Mechatronics, Prague, Czech Republic \\ e-mail: petr.benes@fs.cvut.cz; michael.valasek@fs.cvut.cz
}

\begin{abstract}
The paper deals with the design of optimized input shapers for non-vibrational control of flexible mechatronic systems. The described method is based on a combination of advantages from two approaches - precomputed control curves and on-line shapers. The strategy has two steps. Primarily, an optimized precomputed curve is found as a solution to the point-to-point control problem with respect to any requested optimization goals. Then it is transformed into an on-line shaper with the re-entry property. The resulting shaper transforms any arbitrary input signal to a non-vibrational one. In contrast to other techniques, the shaper length is not determined from the system natural frequency. The shaper can be easily modified with respect to position, velocity, acceleration or jerk control. The theoretical results are verified by experiments using a laboratory crane.
\end{abstract}

Keywords: input shaper, command shaper, residual vibrations, re-entry

\section{Introduction}

The fast and precise positioning of mechatronic structures is a challenging problem when flexibility has to be taken into account and residual vibrations appear. There are basically two main control approaches - feedback and feedforward control. Application of shaped input signals belongs to the latter group. It is based on the input signal that is modified to achieve zero residual vibrations. This principle has been effectively used in many applications such as the robot manipulator (Chang et al., 2005), telescopic handler (Park et al., 2004), antisway crane (Valášek, 1995) etc.

The first form of command/input shaping was the posicast control (Smith, 1957). The step signal was broken into two smaller steps, one delayed in time with respect to the system natural frequency. Superposition of the responses to these steps led to elimination of vibrations. The method is very sensitive to modeling errors. Nevertheless, it was evolved using more step changes (Sugiyama and Uchino, 1986) or precisely timed sine waves (Aspinwall, 1980). Other approaches were based on an analysis using the Laplace transform (domain) synthesis (Bhat and Miu, 1990; Singh and Vadali, 1993) or using the theory of time-delayed systems (Vyhlídal et al., 2012).

Today, we can divide existing shaping techniques into two groups - precomputed curves and on-line shapers (Singhose and Seering, 1997). The former ones solve the point-to-point control problem when both initial and final states of the system are known in advance, but they cannot be used in case the input is changing. Usually, they are based on a combination of precomputed signals with a zero contribution to the system natural frequency and are used, e.g. for design of cam profiles (Wiederrich and Roth, 1974) or in time optimal control (Lau and Pao, 2003). Some of them are based on the differential flatness property of a nonlinear system and enable generation of a trajectory following the control (Post et al., 2011). Shapers, on the other hand, 
act like filters of any arbitrary input signal and modify it not to excite vibration, Fig. 1. The price for that is a delay in the settling time and/or an increase in the required power (Beneš,
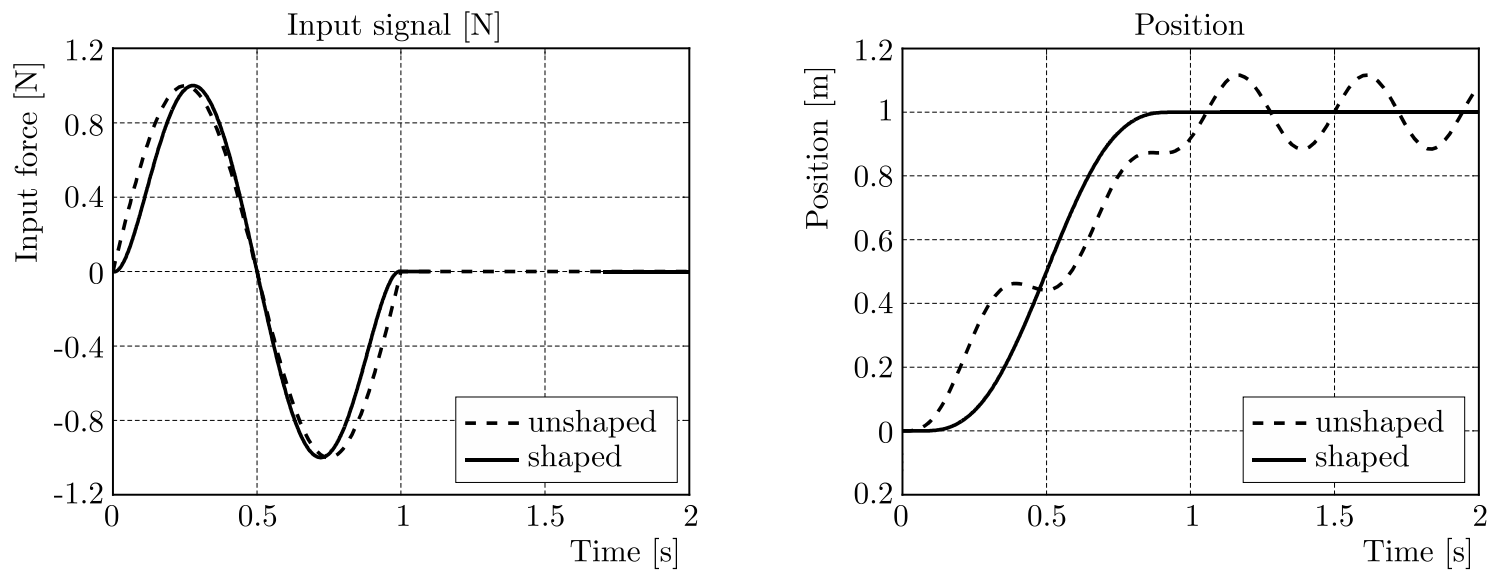

Fig. 1. Comparison of the system response to the shaped and unshaped control input

2012). Shapers are capable of processing a new input signal even if the previous task has not yet been finished. We call this behaviour re-entry property. Probably the most popular method in this group is the patented "Input Shaping" (Singhose, 1997; Singer and Seering, 1990; Singhose and Seering, 1991) based on the convolution of the input signal with a series of precisely timed and scaled pulses.

The advantages and disadvantages of both existing groups are obvious. Precomputed curves can be highly optimised but they do not have the re-entry property and, therefore, cannot be used for on-line systems, e.g. a manually operated crane. Shapers are capable of dealing with on-line control but usually they cannot be optimised to a greater extent because their time length is determined as a multiple of the system natural period and the shape is usually limited to pulses, steps and ramps.

Another problem is that the above mentioned approaches are designed and mostly applied to linear systems. Their extension towards nonlinear systems is a great challenge. The crane is in fact a non-linear system. Many on-line shapers assume that the influence of non-linearity is small and they are successful (Post et al., 2011). The second approach is that non-linear dynamics is decomposed into quasi-linear subsystems. The example is a rotary crane (derrick) decomposed into an equivalent portal crane tangential to the trajectory where separate on-line shapers are used (Zavřel et al., 2004; Piazzi et al., 2002). The third approach is usage of differential flatness that constructs the full relationship between the input and all outputs (Post et al., 2011; Schindele et al., 2009; Zimmert and Sawodny, 2010; Osmic et al., 2014; Heyden and Woernle, 2006). The fourth approach is the use of a non-linear quadratic regulator (NQR) for stable solution of the trajectory (Kittnar et al., 2004). The fifth approach is control using harmonic functions that constructs the missing control input for flexible modes (Neusser et al., 2013). The third to fifth approaches heavily depend on the knowledge of the system model and complete state measurement. If this is not fulfilled, the control quality is deteriorated. The first and second approaches based on shapers show robustness to model uncertainties and are still worth further developing.

Therefore, this paper describes a new approach to the shaper design which combines the advantages of both groups of precomputed curves and on-line re-entry shapers. The completely new result is an optimized on-line shaper with adjustable time length which makes it possible to minimize the required power of the used drives for the chosen reasonable positioning time. 


\section{Theoretical background}

The state space representation of a system with the system matrix $\mathbf{A}$, input matrix $\mathbf{B}$, state vector $\mathbf{y}$ and input vector $\mathbf{u}$ is written as

$$
\dot{\mathbf{y}}=\mathbf{A y}(t)+\mathbf{B u}(t)
$$

The solution to the point-to-point (PTP) control problem for (2.1) with the start time at $t_{1}$, the finish time $t_{2}$ and known initial and final states $\mathbf{y}\left(t_{1}\right), \mathbf{y}\left(t_{2}\right)$, respectively, can be derived in the form (Lewis, 1992)

$$
\mathbf{y}\left(t_{2}\right)=\mathrm{e}^{\mathbf{A}\left(t_{2}-t_{1}\right)} \mathbf{y}\left(t_{1}\right)+\int_{t_{1}}^{t_{2}} \mathrm{e}^{\mathbf{A}\left(t_{2}-\tau\right)} \mathbf{B u}(\tau) d \tau
$$

which can be transformed to

$$
\mathrm{e}^{-\mathbf{A} t_{2}} \mathbf{y}\left(t_{2}\right)-\mathrm{e}^{-\mathbf{A} t_{1}} \mathbf{y}\left(t_{1}\right)=\int_{t_{1}}^{t_{2}} \mathrm{e}^{-\mathbf{A} \tau} \mathbf{B} \mathbf{u}(\tau) d \tau
$$

The expression on the right side of (2.4) can be rewritten as a sum of contributions from particular inputs

$$
\mathrm{e}^{-\mathbf{A} t_{2}} \mathbf{y}\left(t_{2}\right)-\mathrm{e}^{-\mathbf{A} t_{1}} \mathbf{y}\left(t_{1}\right)=\sum_{l=1}^{n} \int_{t_{1}}^{t_{2}} \mathrm{e}^{-\mathbf{A} \tau} u_{l}(\tau) d \tau \mathbf{b}_{l}
$$

where $n$ is the total number of inputs, $u_{l}$ is the $l$-th input, $\mathbf{b}_{l}$ is the corresponding column of the B matrix.

Assuming the controllability of system (2.1), there is a unique transform to the Jordan canonical form

$$
\dot{\mathbf{z}}(t)=\mathbf{J} \mathbf{z}(t)+\mathbf{D u}(t) \quad \mathbf{J}=\mathbf{P}^{-1} \mathbf{A} \mathbf{P} \quad \mathbf{z}=\mathbf{P}^{-1} \mathbf{y} \quad \mathbf{D}=\mathbf{P}^{-1} \mathbf{B}
$$

where $\mathbf{J}$ is a block diagonal matrix made of Jordan blocks, $\mathbf{P}$ is a regular transform matrix. Then the solution to (2.3) can be expressed as

$$
\mathrm{e}^{-\mathbf{J} t_{2}} \mathbf{z}\left(t_{2}\right)-\mathrm{e}^{-\mathbf{J} t_{1}} \mathbf{z}\left(t_{1}\right)=\int_{t_{1}}^{t_{2}} \mathrm{e}^{-\mathbf{J} \tau} \mathbf{D} \mathbf{u}(\tau) d \tau
$$

with

$$
\mathrm{e}^{\mathbf{J} t}=\operatorname{diag}\left\{\mathrm{e}^{\mathbf{J}_{i} t}\right\} \quad \mathrm{e}^{\mathbf{J}_{i} t}=\mathrm{e}^{p_{i} t}\left[\begin{array}{ccccc}
1 & 0 & \cdots & 0 & 0 \\
t & 1 & \cdots & 0 & 0 \\
\vdots & \vdots & \ddots & \vdots & \vdots \\
\frac{t^{r_{i}-1}}{\left(r_{i}-1\right) !} & \frac{t^{r_{i}-2}}{\left(r_{i}-2\right) !} & \cdots & 1 & 0 \\
\frac{t^{r_{i}}}{r_{i} !} & \frac{t^{r_{i}-1}}{\left(r_{i}-1\right) !} & \cdots & t & 1
\end{array}\right]
$$

where $p_{i}$ is the pole of the Jordan block $\mathbf{J}_{i}$ with the order $r_{i}+1$. Analogously to (2.4), the right side of (2.6) can be rewritten as a sum of particular inputs $u_{l}$

$$
\mathrm{e}^{-\mathbf{J} t_{2}} \mathbf{z}\left(t_{2}\right)-\mathrm{e}^{-\mathbf{J} t_{1}} \mathbf{z}\left(t_{1}\right)=\sum_{l=1}^{n} \int_{t_{1}}^{t_{2}} \mathrm{e}^{-\mathbf{J} \tau} u_{l}(\tau) d \tau \mathbf{d}_{l}
$$

where $\mathbf{d}_{l}$ is the $l$-th column of $\mathbf{D}$ matrix corresponding to the input $u_{l}$. 
Note that the convolution integral on the right side resembles the finite time Laplace transform as defined by Miu (1993)

$$
U(s)=\int_{t_{1}}^{t_{2}} \mathrm{e}^{-s \tau} u(\tau) d \tau
$$

therefore, the solution to the PTP control problem can be written as

$$
\left.\sum_{l=1}^{n} U_{l}(s)\right|_{s=\mathbf{J}} \mathbf{d}_{l}=\mathrm{e}^{-\mathbf{J} t_{2}} \mathbf{z}\left(t_{2}\right)-\mathrm{e}^{-\mathbf{J} t_{1}} \mathbf{z}\left(t_{1}\right)
$$

or more generally for the system not in the Jordan form

$$
\left.\sum_{l=1}^{n} U_{l}(s)\right|_{s=\mathbf{A}} \mathbf{b}_{l}=\mathrm{e}^{-\mathbf{A} t_{2}} \mathbf{y}\left(t_{2}\right)-\mathrm{e}^{-\mathbf{A} t_{1}} \mathbf{y}\left(t_{1}\right)
$$

where $U_{l}(s)$ is the finite time Laplace transform of the $l$-th input. The solution $u_{l}(t)$ in the time domain is the inverse finite time Laplace transform of $U_{l}(s)$.

Note that general equation (2.11) enables calculation of the control input for any arbitrary boundary states $\mathbf{y}\left(t_{1}\right), \mathbf{y}\left(t_{2}\right)$. The rest-to-rest positioning without vibrations in the final position is just one special application. Another application could be, e.g. smooth acceleration to a desired velocity or even vibration elimination from a known non-zero initial state. Moreover, conditions (2.11) are algebraic and, therefore, there is no need to solve differential equations. Finally, note that there is no limitation in terms of the time length. It is not necessarily defined by a multiple of the system natural frequency or by other constraints.

Now this approach will be applied to a simple model consisting of two masses $m_{1}, m_{2}$ connected by a spring $k$ and a viscous damper $c$ as shown in Fig. 2.

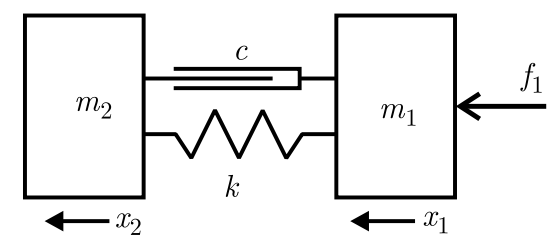

Fig. 2. Two-mass model

This system is described by the matrix equation

$$
\mathbf{M} \ddot{\mathbf{x}}(t)+\mathbf{C} \dot{\mathbf{x}}+\mathbf{K x}(t)=\mathbf{f}(x)
$$

where $\mathbf{x}$ is a vector of coordinates, $\mathbf{M}, \mathbf{C}, \mathbf{K}$ are mass, damping and stiffness matrices, and $\mathbf{f}$ is a vector of input forces (see Fig. 2) defined as

$$
\begin{array}{lc}
\mathbf{x}=\left[\begin{array}{l}
x_{1} \\
x_{2}
\end{array}\right] \quad \mathbf{M}=\left[\begin{array}{cc}
m_{1} & 0 \\
0 & m_{2}
\end{array}\right] \quad \mathbf{C}=\left[\begin{array}{cc}
c & -c \\
-c & c
\end{array}\right] \\
\mathbf{K}=\left[\begin{array}{cc}
k & -k \\
-k & k
\end{array}\right] \quad \mathbf{f}=\left[\begin{array}{c}
f_{1} \\
0
\end{array}\right] \ldots
\end{array}
$$

Non-vibrational conditions in the final position $X_{f}$ of the PTP task are

$$
\mathbf{x}\left(t_{2}\right)=\left[\begin{array}{l}
X_{f} \\
X_{f}
\end{array}\right] \quad \dot{\mathbf{x}}\left(t_{2}\right)=\left[\begin{array}{l}
0 \\
0
\end{array}\right]
$$


Differential equation of the second order (2.12) can be rewritten as a set of first order equations transformed to the Jordan canonical form and rewritten as

$$
\underbrace{\left[\begin{array}{l}
\dot{y}_{1} \\
\dot{y}_{2} \\
\dot{y}_{3} \\
\dot{y}_{4}
\end{array}\right]}_{\dot{\mathbf{y}}_{1}}=\underbrace{\left[\begin{array}{llll}
0 & 1 & 0 & 0 \\
0 & 0 & 0 & 0 \\
0 & 0 & p & 0 \\
0 & 0 & 0 & p^{*}
\end{array}\right]}_{\mathbf{A}} \underbrace{\left[\begin{array}{l}
y_{1} \\
y_{2} \\
y_{3} \\
y_{4}
\end{array}\right]}_{\mathbf{y}}+\underbrace{\left[\begin{array}{l}
0 \\
1 \\
1 \\
1
\end{array}\right]}_{\mathbf{B}} u
$$

where $u=f_{1}\left(m_{1}+m_{2}\right) /\left(m_{1} m_{2}\right)$ and $p, p^{*}$ are complex conjugated poles of flexible modes

$$
\begin{array}{ll}
p=-\xi \omega+j \sqrt{1-\xi^{2}} \omega & p^{*}=-\xi \omega-j \sqrt{1-\xi^{2}} \omega \\
\omega & =\sqrt{\frac{k\left(m_{1}+m_{2}\right)}{m_{1} m_{2}}} \quad \xi=\frac{c\left(m_{1}+m_{2}\right)}{2 \omega m_{1} m_{2}}
\end{array}
$$

Actually, the use of the Jordan form is not necessary, but here it is used for a better understating of the whole calculation process and also for the proof of the formula of Bhat and Miu (1991).

Boundary conditions (2.14) are transformed to

$$
\mathbf{y}\left(t_{2}\right)=\left[X_{f}, 0,0,0\right]^{\mathrm{T}}
$$

The general solution to the PTP control problem is defined by equation (2.11). Assuming that $t_{1}=0, t_{2}=T$ and zero initial conditions $\mathbf{y}\left(t_{1}\right)=\mathbf{0}$, the right side of $(2.11)$ is

$$
\begin{gathered}
\mathrm{e}^{-\mathbf{A} t_{2}} \mathbf{y}\left(t_{2}\right)-\mathrm{e}^{-\mathbf{A} t_{1}} \mathbf{y}\left(t_{1}\right)=\mathrm{e}^{-\mathbf{A} T} \mathbf{y}(T)-\mathrm{e}^{-\mathbf{A} 0} \mathbf{y}(0)=\mathrm{e}^{(-\mathbf{A} T)}\left[\begin{array}{c}
X_{f} \\
0 \\
0 \\
0
\end{array}\right]-\mathrm{e}^{(-\mathbf{A} 0)}\left[\begin{array}{l}
0 \\
0 \\
0 \\
0
\end{array}\right] \\
=\left[\begin{array}{cccc}
1 & -T & 0 & 0 \\
0 & 1 & 0 & 0 \\
0 & 0 & \mathrm{e}^{-p T} & 0 \\
0 & 0 & 0 & \mathrm{e}^{-p^{*} T}
\end{array}\right]\left[\begin{array}{c}
X_{f} \\
0 \\
0 \\
0
\end{array}\right]=\left[\begin{array}{c}
X_{f} \\
0 \\
0 \\
0
\end{array}\right]
\end{gathered}
$$

The expression on the left side in (2.11) can be calculated as follows. Let us assume that the finite time Laplace transform $U(s)$ can be written as a Taylor series

$$
U(s)=U_{0}+U_{1} s+U_{2} s^{2}+\ldots
$$

then

$$
\begin{aligned}
& \left.U(s)\right|_{s=\mathbf{A}}=U_{0}\left[\begin{array}{cccc}
1 & 0 & 0 & 0 \\
0 & 1 & 0 & 0 \\
0 & 0 & 1 & 0 \\
0 & 0 & 0 & 1
\end{array}\right]+U_{1}\left[\begin{array}{cccc}
0 & 1 & 0 & 0 \\
0 & 0 & 0 & 0 \\
0 & 0 & p & 0 \\
0 & 0 & 0 & p^{*}
\end{array}\right]+U_{2}\left[\begin{array}{cccc}
0 & 0 & 0 & 0 \\
0 & 0 & 0 & 0 \\
0 & 0 & p^{2} & 0 \\
0 & 0 & 0 & p^{* 2}
\end{array}\right]+\ldots \\
& \left.U(s)\right|_{s=\mathbf{A}}=\left[\begin{array}{cccc}
U_{0} & U_{1} & 0 & 0 \\
0 & U_{0} & 0 & 0 \\
0 & 0 & U_{0}+U_{1} p+U_{2} p^{2}+\ldots & 0 \\
0 & 0 & 0 & U_{0}+U_{1} p^{*}+U_{2} p^{* 2}+\ldots
\end{array}\right]
\end{aligned}
$$


According to (2.11) $U(s)$ is multiplied by the input vector $\mathbf{b}_{1}$ (note that there is only one input)

$$
\begin{aligned}
& \left.U(s)\right|_{s=\mathbf{A}}[0 ; 1 ; 1 ; 1]^{\mathrm{T}}=\left[\begin{array}{cccc}
U_{0} & U_{1} & 0 & 0 \\
0 & U_{0} & 0 & 0 \\
0 & 0 & U_{0}+U_{1} p+U_{2} p^{2}+\ldots & 0 \\
0 & 0 & 0 & U_{0}+U_{1} p^{*}+U_{2} p^{* 2}+\ldots
\end{array}\right] \\
& =\left[\begin{array}{c}
U_{1} \\
U_{0} \\
U_{0}+U_{1} p+U_{2} p^{2}+\ldots \\
U_{0}+U_{1} p^{*}+U_{2} p^{* 2}+\ldots
\end{array}\right]=\left[\begin{array}{c}
\left.\frac{d U(s)}{d s}\right|_{s=0} \\
U(0) \\
U(p) \\
U\left(p^{*}\right)
\end{array}\right]
\end{aligned}
$$

Using (2.18) and (2.21), the conditions for non-vibrational control input (2.11) can be formulated in a component form as

$$
\left.\frac{d U(s)}{d s}\right|_{s=0}=\left.X_{f} \quad U(s)\right|_{s=0}=\left.0 \quad U(s)\right|_{s=p}=\left.0 \quad U(s)\right|_{s=p^{*}}=0
$$

The simple analytical formulation of necessary conditions for non-vibrational control (2.22) used by Bhat and Miu (1991) is the result of description of the system in a canonical form. Other state space representations usually need a numerical solution of (2.11). According to the authors' best knowledge, the step-by-step derivation of these conditions presented here has not been published before.

Note that unlike other methods (Smith, 1957; Aspinwall, 1980; Sugiyama and Uchino, 1986) using this formulation, the time length of the input signal $T$ is not strictly defined as a multiple of the system natural period and can be set arbitrarily. However, the solution $u(t)$ from $(2.22)$ must be precomputed before the PTP operation and during the operation cannot be modified as it is often required (see Section 6).

\section{Control input synthesis}

There is an infinite number of functions $u(t)$ which satisfy equations (2.22). An algebraic method for the synthesis of the control input called the Laplace transform (domain) synthesis technique (Miu, 1993) assumes the control input as a linear combination of independent basis functions $\phi_{i}(t)$ multiplied by weighting coefficients $\lambda_{i}$

$$
u(t)=\sum_{i=1}^{2 q+2} \lambda_{i} \phi_{i}(t)
$$

where $q$ is a number of flexible modes pairs. Then, according to (2.22)

$$
\begin{aligned}
& {\left[\left.\frac{d U(s)}{d s}\right|_{s=0},\left.U(s)\right|_{s=0},\left.U(s)\right|_{s=p},\left.U(s)\right|_{s=p^{*}}\right]^{\mathrm{T}}=\mathbf{S} \boldsymbol{\lambda}=\underbrace{\left[X_{f}, 0, \ldots, 0\right]^{\mathrm{T}}}_{\mathbf{y}(T)}} \\
& \mathbf{S}=\left[\begin{array}{ccc}
\frac{d \Phi_{1}(0)}{d s} & \cdots & \frac{d \Phi_{2 q+2}(0)}{d s} \\
\Phi_{1}(0) & \cdots & \Phi_{2 q+2}(0) \\
\Phi_{1}\left(p_{1}\right) & \cdots & \Phi_{2 q+2}\left(p_{1}\right) \\
\vdots & \ddots & \vdots \\
\Phi_{1}\left(p_{q}^{*}\right) & \cdots & \Phi_{2 q+2}\left(p_{q}^{*}\right)
\end{array}\right]
\end{aligned}
$$


where $\boldsymbol{\lambda}=\left[\lambda_{1}, \lambda_{2}, \ldots, \lambda_{2 q+2}\right]^{\mathrm{T}}$ and $\Phi_{i}(s)$ are finite time Laplace transforms of the basis functions $\phi_{i}$.

Using (3.2) 1 , the weighting coefficients $\boldsymbol{\lambda}$ can be obtained as

$$
\boldsymbol{\lambda}=\mathbf{S}^{-1}\left[X_{f}, 0, \cdots, 0\right]^{\mathrm{T}}
$$

But such formulation does not take into account specific properties of the chosen basis functions $\phi_{i}(t)$. Some choices lead to non-invertible matrix $\mathbf{S}$, in some cases the solution does not exist (Beneš, 2012).

The solution to $(3.2)_{1}$ exists if the rank $h$ of matrix $\mathbf{S}$ is equal to the rank of the augmented matrix $\left[\mathbf{S} \mid \mathbf{y}\left(t_{2}\right)\right]$

$$
h=h(\mathbf{S})=h\left(\mathbf{S} \mid \mathbf{y}\left(t_{2}\right)\right)
$$

Note that $h(\mathbf{S})$ is not necessarily equal to $2 q+2$ from (3.1) because the rows of $\mathbf{S}$ could be linear dependent.

Therefore, instead of (3.1) the control input should be reformulated to the form

$$
u(t)=\sum_{i=1}^{j} \lambda_{i} \phi_{i}(t) \quad j \geqslant h
$$

In the case $j=h$, there is one and only one combination of weighting coefficients $\lambda_{i}$, in the case $j>h$ the infinite number of solutions exists. For $j<h$, the solution to (2.22) does not exist and $j$ must be increased.

Equation (3.2) $)_{1}$ ensures that no vibration appears in PTP positioning. To meet other criteria, more constrains could be added to this synthesis technique. These constrains can be formulated both in s-domain and time domain. The common one is e.g. the time-domain continuity constraint (Miu, 1993), but this could be fulfilled using a proper set of basis functions, see (4.2). Additional constraints can be used, e.g. for specifying states between the initial and final time (trajectory tracking) or to increase robustness of the control to modelling errors. The increasing of the robustness is based on placing multiple zeros in the system poles or on placing new zeros near to the system poles. This strategy is similar to the idea of ZVD or EI shapers in Singhose (1997), but as the time length can be still set arbitrarily this does not mean prolongation of the input signal. Instead of an increase of the necessary time, we can increase the power used for positioning. Or, probably more often, we can find the right balance between the signal length and the required power regardless of the system natural frequency.

\section{Optimization using free weighting coefficients}

The situation $j>h$ in (3.5) means that the first $h$ coefficients can be expressed using nonvibrational conditions (2.22) as a function of the rest of them, $\lambda_{i}=f_{i}\left(\lambda_{h+1}, \ldots, \lambda_{j}\right), i=1, \ldots, h$. Then the free coefficients $\lambda_{h+1}, \ldots, \lambda_{j}$ could be used to meet additional conditions or as optimization parameters. This will be demonstrated using the simple model in Fig. 2. The optimization goal is minimization of the required maximum power of the drive. Therefore, the cost function for minimization is defined as

$$
f_{c}=\max |u(t)| \quad t \in\left\langle 0, t_{2}\right\rangle
$$


Consider the control input as a linear combination of sine waves in the form

$$
\begin{aligned}
& u(t)=\sum_{i=1}^{2 q+2} \lambda_{i} \phi_{i}(t)=\sum_{i=1}^{4} \lambda_{i} \sin \left(\omega_{s} i t\right) \\
& U(s)=\sum_{i=1}^{2 q+2} \lambda_{i} \Phi_{i}(s)=\sum_{i=1}^{4} \lambda_{i} \frac{\mathrm{e}^{-s t_{2}}\left(-s \sin \left(\omega_{s} t_{2}\right)-\omega \cos \left(\omega_{s} t_{2}\right)\right)+\omega_{s}}{s^{2}+\omega_{s}^{2}}
\end{aligned}
$$

with $\omega_{s}=2 \pi / t_{2}$, which ensures the time domain continuity requirement automatically. The supposed system parameters are $m_{1}=m_{2}=1 \mathrm{~kg}, k=100 \mathrm{~kg} \cdot \mathrm{s}^{-2}, c=0 \mathrm{~kg} \cdot \mathrm{s}^{-1}$. The desired rigid body displacement is $X_{\mathrm{f}}=1 \mathrm{~m}$ and $t_{2}=1 \mathrm{~s}$. Then the matrix $\mathbf{S}$ is

$$
\mathbf{S}=\left[\begin{array}{cccc}
0.1592 & 0.0796 & 0.0531 & 0.0398 \\
0 & 0 & 0 & 0 \\
-0.0393-0.0391 \mathrm{i} & -0.3001-0.2986 \mathrm{i} & 0.1220+0.1214 \mathrm{i} & 0.0585+0.0582 \mathrm{i} \\
-0.0393+0.0391 \mathrm{i} & -0.3001+0.2986 \mathrm{i} & 0.1220-0.1214 \mathrm{i} & 0.0585-0.0582 \mathrm{i}
\end{array}\right]
$$

and it has $\operatorname{rank} h(\mathbf{S})=2$. Since $j=4$, we have two free parameters for optimization. Using $(3.2)_{1}$, we obtain

$$
\lambda_{1}=6.7239-0.5742 \lambda_{3}-0.3719 \lambda_{4} \quad \lambda_{2}=-0.8815+0.4818 \lambda_{3}+0.2437 \lambda_{4}
$$

The basic non-optimal solution is that with $\lambda_{3}=\lambda_{4}=0$

$$
\lambda_{\text {bas }}=[6.7239,-0.8815,0,0]^{\mathrm{T}}
$$

Simple optimization of the parameters $\lambda_{i}$ according to cost function (4.1) by e.g. fminsearch in the Matlab environment results in

$$
\boldsymbol{\lambda}_{\text {opt }}=[5.6154,0.0273,1.7280,0.3127]^{\mathrm{T}}
$$

The plots for a standard basic solution (4.5) and optimized solution (4.6) are compared in Fig. 3. The required power is reduced by about $25 \%$.
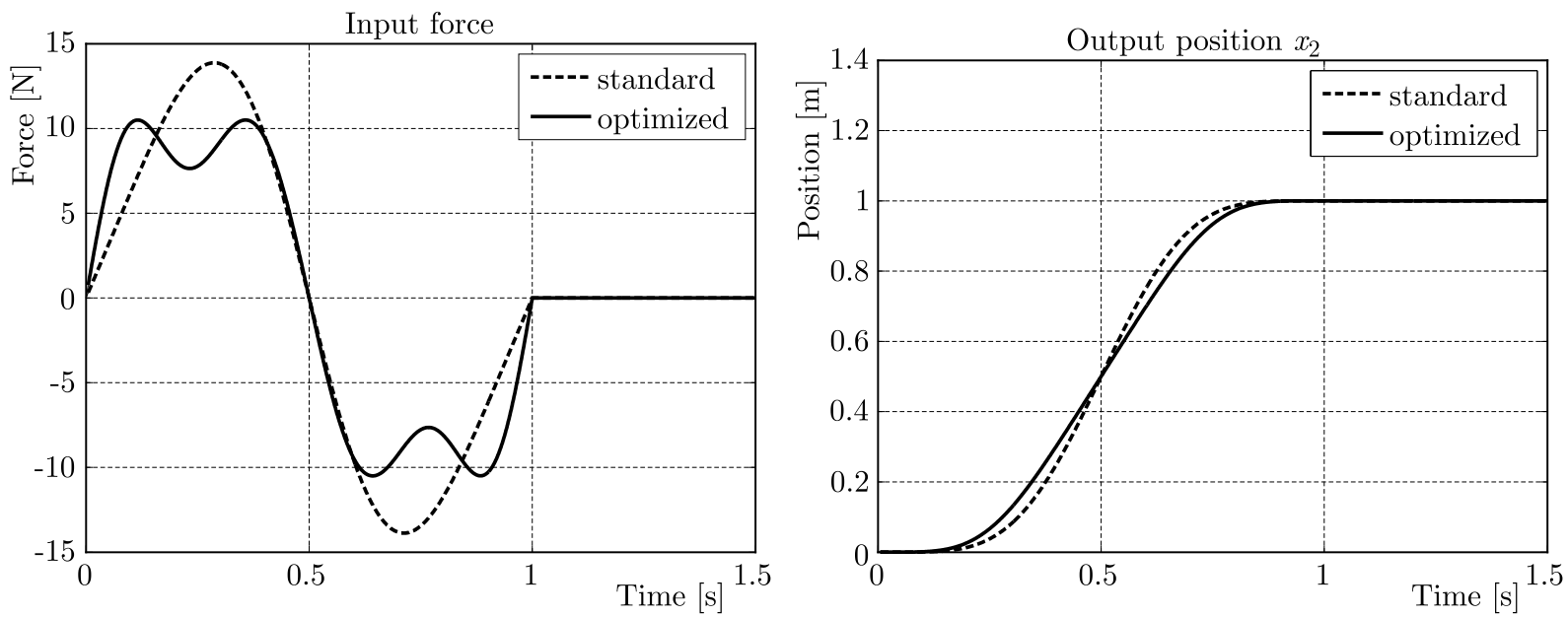

Fig. 3. System response to the basic and optimized shape of the input force

There could be more than one optimization goal. Figure 4 shows a Pareto set when both the required power and its derivative are minimized. The minimized derivative of the required power is important for reduction of costs of power electronics. The depicted points represent various combinations of $\lambda_{3}$ and $\lambda_{4}$. Other optimization criteria can be formulated, e.g. to increase the robustness to model errors, etc. 


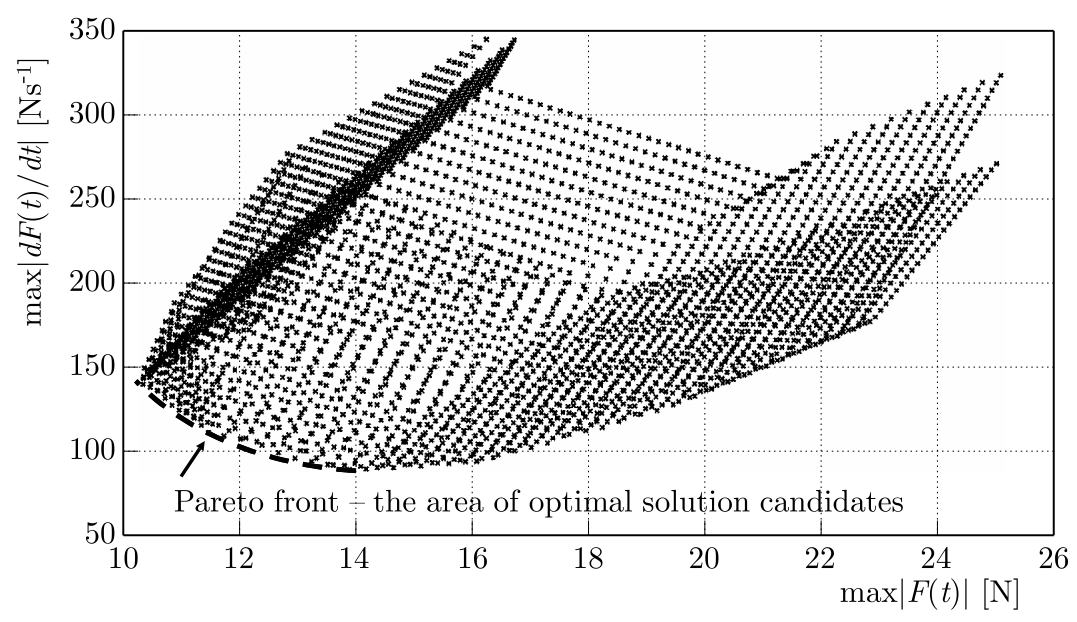

Fig. 4. Pareto set

\section{Optimization using parametric basis functions}

Besides searching for an optimal combination of weighting coefficients, different parametric basis functions can be used. In such a case, the optimization is focused on these parameters. A control function generated as a combination of damped sinusoids is used as an example

$$
u(t)=\lambda_{1} \mathrm{e}^{a_{1} t} \sin \left(\frac{2 \pi}{T} t\right)+\lambda_{2} \mathrm{e}^{a_{2} t} \sin \left(\frac{4 \pi}{T} t\right)+\lambda_{3} \mathrm{e}^{a_{3} t} \sin \left(\frac{6 \pi}{T} t\right)+\lambda_{4} \mathrm{e}^{a_{4} t} \sin \left(\frac{8 \pi}{T} t\right)
$$

where the coefficients $a_{i}<0$ are the optimization parameters. The goal of the optimization is minimization of the required power.

The optimization leads to the following coefficients

$$
\mathbf{a}=[-0.0164,-37.9986,-0.0001,-42.3485]^{\mathrm{T}}
$$

with the corresponding

$$
\lambda_{\text {opt }}=[5.9175,-101.8635,1.1877,75.6287]^{\mathrm{T}}
$$

The results are compared in Fig. 5 with basic non-optimal solution (4.5) which corresponds to the setting $a_{1,2,3,4}=0$. The required power is reduced by about $28 \%$, which is even slightly better than that in Fig. 3 .
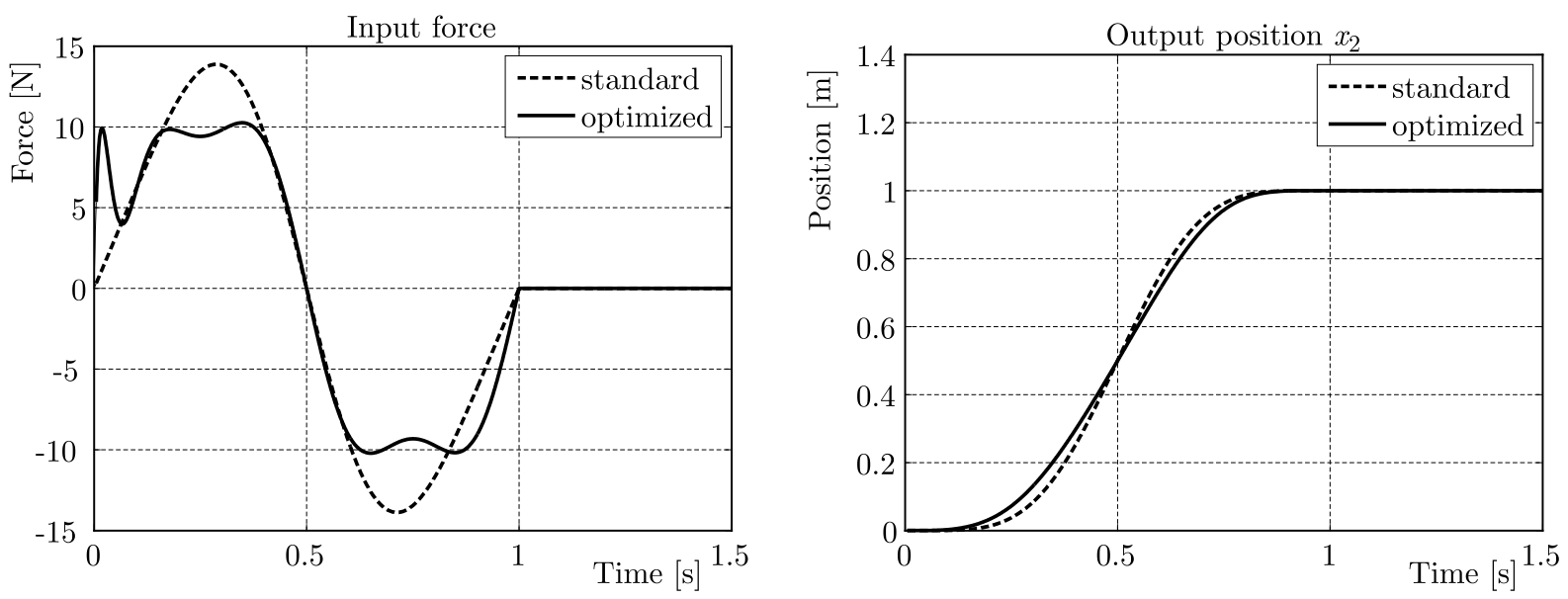

Fig. 5. System response to the parametrically optimized shape of the input force 


\section{Re-entry online shaper from a precomputed curve}

An approach described in previous Sections produces control inputs in the form of precomputed curves. But these are not applicable when the system must be controlled on-line and the final state is not known in advance. This could be for example a crane manually controlled by a human operator. In such a case, we need a shaper which transforms any arbitrary input signal to a non-vibrational one (Beneš et al., 2008). These shapers are usually based on the patented "Input Shaping" (Singhose, 1997; Singer and Seering, 1990; Singhose and Seering, 1991) series of pulses with time length fixed to the system natural frequency or even its multiples. Now a more general technique will be described, which transforms an optimized control curve with an arbitrarily set time length into a dynamic shaper.

The new approach (Beneš, 2012; Beneš et al., 2008) for such cases is as follows. The general conditions for a control signal which does not excite vibrations are formulated in Section 2. Now the signal which fulfils these conditions should be a product of the convolution of any arbitrary input signal with the shaper. If the input signal is a unit pulse then the product of convolution should be of the same shape as the corresponding precomputed curve, as shown in Fig. 6. In other words, the shaper is a dynamic representation of a non-vibrational control curve. If the control curve transforms the system from a zero initial state to a unit final state then we obtain a shaper with the unit gain. The signal modified by this shaper transforms the system to the same final state as the unshaped one but with zero residual vibrations. The procedure to synthetize such a shaper is to find a differential equation and a corresponding dynamic block the solution to which is the non-vibrational control curve from Section 2. This is a so called re-entry shaper, and it can be re-entered during operation.

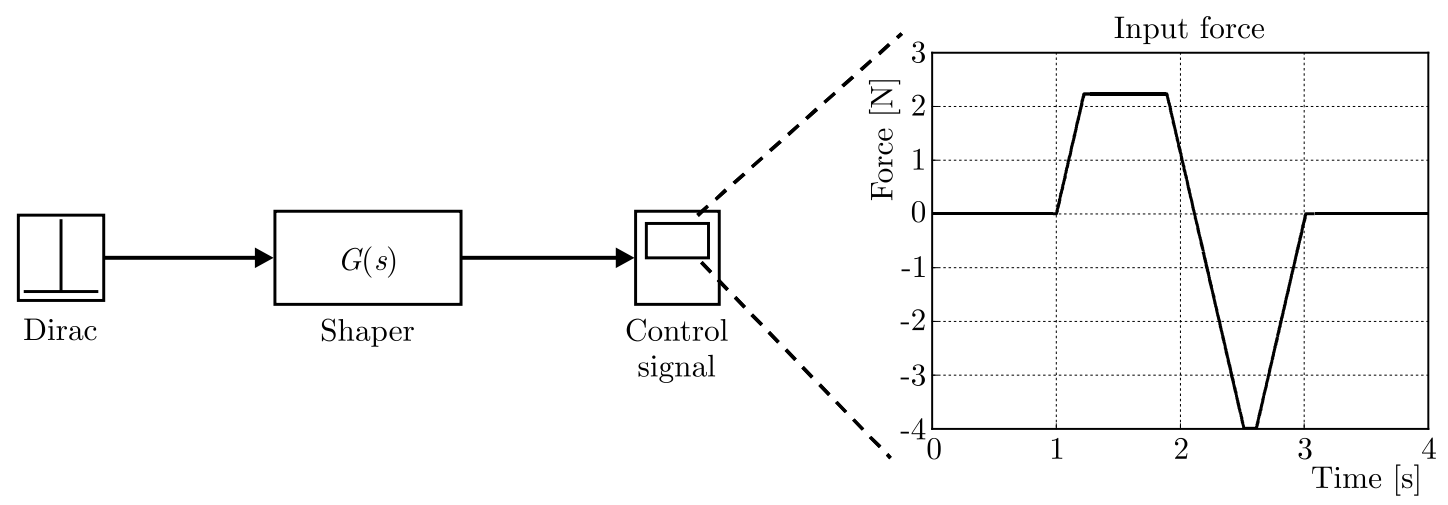

Fig. 6. Shaper convolution with the Dirac pulse

It is difficult to find a dynamic block corresponding to a certain general curve. Consequently, it is a great benefit that our control signal is a linear combination of the basis functions. The dynamic representation of the basis function is actually its finite time Laplace transform. For example, the ramp shaper used in Fig. 6 is described as a transfer function

$$
U(s)=\frac{q}{s^{2}}\left(1-\mathrm{e}^{-s t_{1}}-\mathrm{e}^{-s t_{2}}+\mathrm{e}^{-s t_{3}}+\mathrm{e}^{-s t_{4}}-\mathrm{e}^{-s t_{5}}\right)
$$

where $q$ is the ramp angle, $t_{i}$ are the switching times. The shape of the control curve is shown in Fig. 7.

As the positive and negative parts of the ramp must be of the same length, the $t_{5}$ is defined as

$$
t_{5}=t_{4}+t_{3}-t_{2}-t_{1}
$$




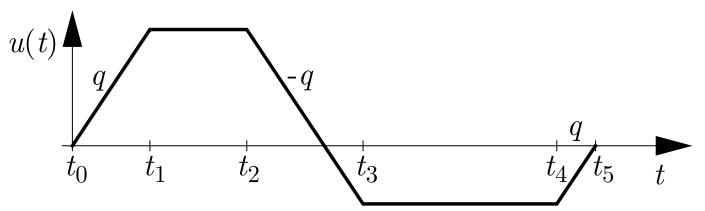

Fig. 7. Shape of the ramp control curve

Function (6.1) can be rewritten in a more compact form as

$$
U(s)=\frac{q}{s^{2}} \sum_{i=0}^{5} N_{i} \mathrm{e}^{-s t_{i}}
$$

with

$$
\mathbf{N}=[1,-1,-1,1,1,-1] \quad t_{0}=0
$$

For the two-mass model in Fig. 2 with the parameters $m_{1}=m_{2}=1 \mathrm{~kg}, k=100 \mathrm{~kg} \cdot \mathrm{s}^{-2}$, $c=1 \mathrm{~kg} \cdot \mathrm{s}^{-1}$ the non-vibrational conditions are (2.22). Applying these conditions to (6.3), we obtain the following set of conditions

$$
\begin{array}{rlrl}
\sum_{i=0}^{5} N_{i} t_{i}=0 & \sum_{i=0}^{5} N_{i} t_{i}^{2}=0 & & \sum_{i=0}^{5} N_{i} t_{i}^{3}=-\frac{6 X_{f}}{q} \\
\sum_{i=0}^{5} N_{i} \mathrm{e}^{\xi \omega t_{i}} \cos \left(\sqrt{1-\xi^{2}} \omega t_{i}\right)=0 & & \sum_{i=0}^{5} N_{i} \mathrm{e}^{\xi \omega t_{i}} \sin \left(\sqrt{1-\xi^{2}} \omega t_{i}\right)=0
\end{array}
$$

Two of them, $(6.5)_{1}$ and $(6.5)_{2}$, result from $\left.U(s)\right|_{s=0}=0$. According to the definition of $t_{5}$ in equation (6.2), constraint $(6.5)_{1}$ is fulfilled automatically and, therefore, we have a set of four equations $(6.5)_{1}-(6.5)_{5}$ with four unknowns $t_{1}, \ldots, t_{4}$. For $q=5 \mathrm{~m} \cdot \mathrm{s}^{-3}$, the solution is a vector of the switching times

$$
\mathbf{t}=[0,0.2228,0.8922,1.5136,1.6136,2.0123]
$$

Note that due to transformation between the input $u(t)$ and the control force $f_{1}(t)$, $u=f_{1}\left(m_{1}+m_{2}\right) / m_{1} m_{2}$, the defined value of $q$ is a ramp with the slope $q_{F}=10 \mathrm{~N} \cdot \mathrm{s}^{-1}$.

Equation (6.3) can be used for the direct design of a re-entry shaper (Fig. 8). Transfer function (6.3) is actually the description of the differential equation which reproduces the precomputed curve. In this case, it is the ramp shown in Fig. 7.

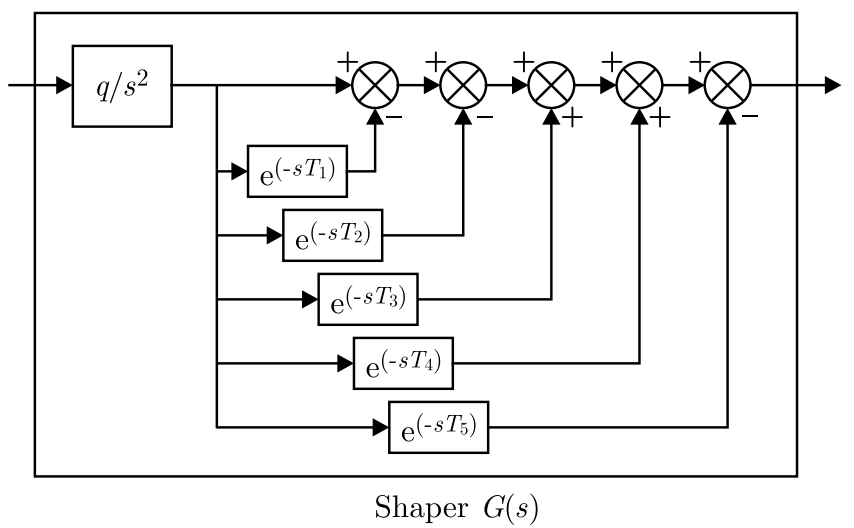

Fig. 8. Re-entry shaper $G(s)$, here $T_{i}=t_{i}$ 

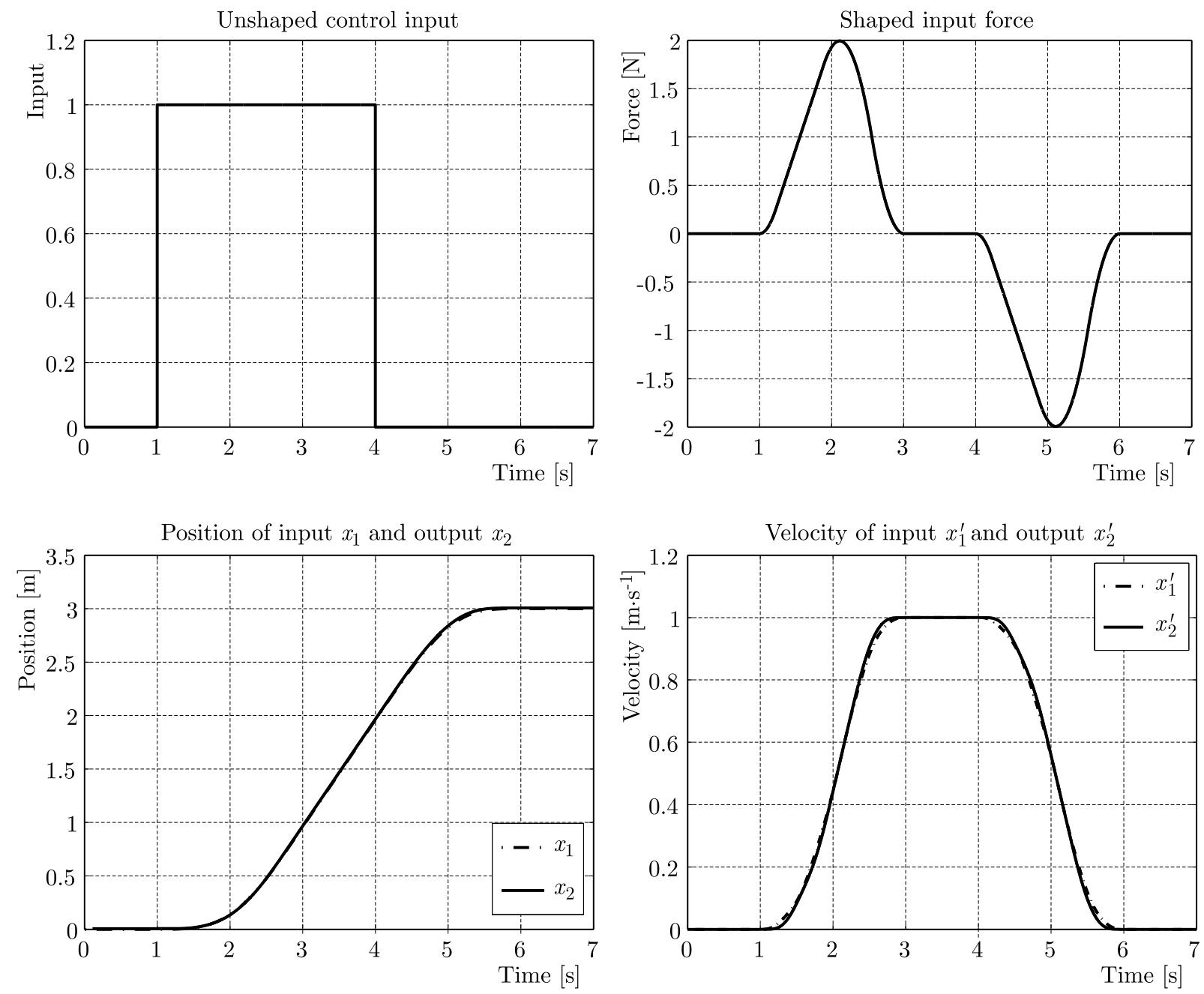

Fig. 9. Re-entry shaper - velocity control

The shaper created in this way represents velocity control, see Fig. 9. But it can be easily transformed to control position, acceleration or jerk. The only modification which has to be done is the change of the power of $s$ in (6.1). If the denominator before the bracket is $s^{3}$ then it acts like acceleration control. If it is changed to $s^{4}$ then we have jerk control. Finally, if it is only $s^{1}$ then it acts like direct position control.

Note that we can use all methods described in the previous Sections, especially optimizations, to design the best precomputed curve with respect to requested criteria. For example, the ramp control function with limited both the slope and maximum value could be prepared to avoid actuator saturation (Beneš, 2012). Then, using the finite time Laplace transform we can change it to the form of the re-entry shaper.

This possibility to synthetize re-entry shapers of prescribed time lengths or optimized to chosen goals is the main contribution of this paper. It eliminates the drawbacks of both previous approaches (Bhat and Miu, 1990; Singhose, 1997).

\section{Experiment - antisway crane}

An antisway crane is one of the typical benchmarks for tests of non-vibration control strategies. The laboratory model shown in Fig. 10 is controlled by simple buttons with 2 states (on/off). But the rectangular input that goes from the buttons excites vibrations of the load. The goal 


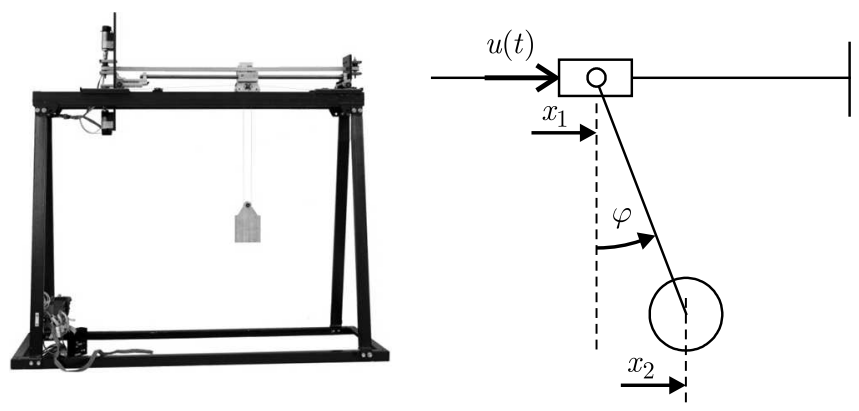

Fig. 10. Crane - a laboratory model and its diagram

is to design an on-line shaper which modifies the signal from the buttons to a non-vibrational one.

According to the approach described in previous Sections primarily the optimized control curve is calculated and then it is transformed using the finite time Laplace transform to an on-line shaper. The shape of the control curve in parametrical form (5.1) is used. The optimization is focused on settling time minimization with respect to the crane model parameters.

During the tests, the model was manually controlled by a human operator who performed a set of various manoeuvres with varied cable length as well. The results of one of them are shown in Fig. 11 (unshaped) and in Fig. 12 (shaped). The swinging in the final position was almost completely eliminated when the shaper was used.
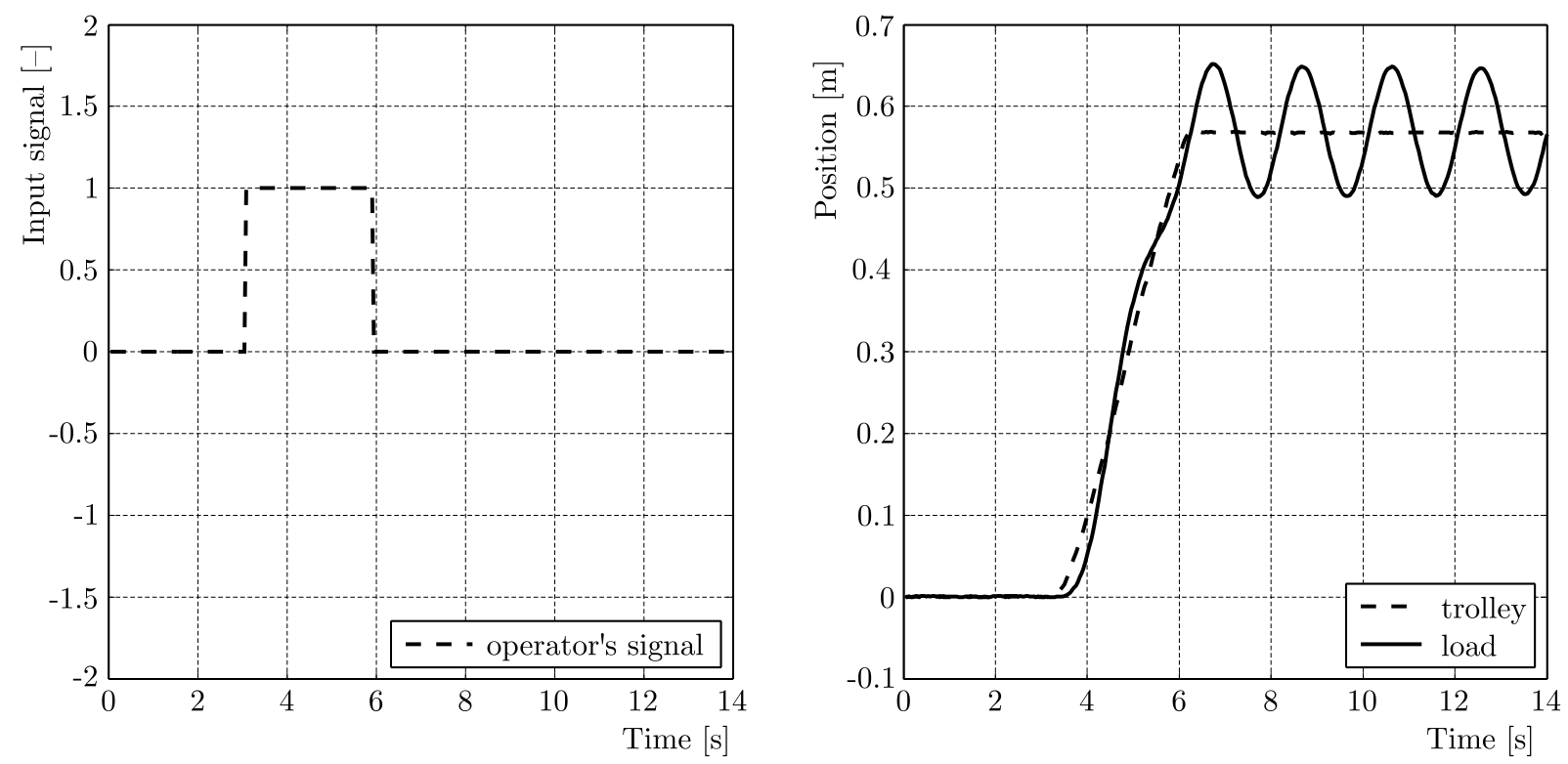

Fig. 11. Experiment - the system response without the shaper

Another test was focused on the re-entry property of the shaper. The operator randomly switched between the right and left direction of the trolley without waiting for the end of the previous manoeuver. The results for unshaped control are given in Fig. 13a and for control with the shaper in Fig. 13b. Again, the swinging in the final position was eliminated by the shaper. 

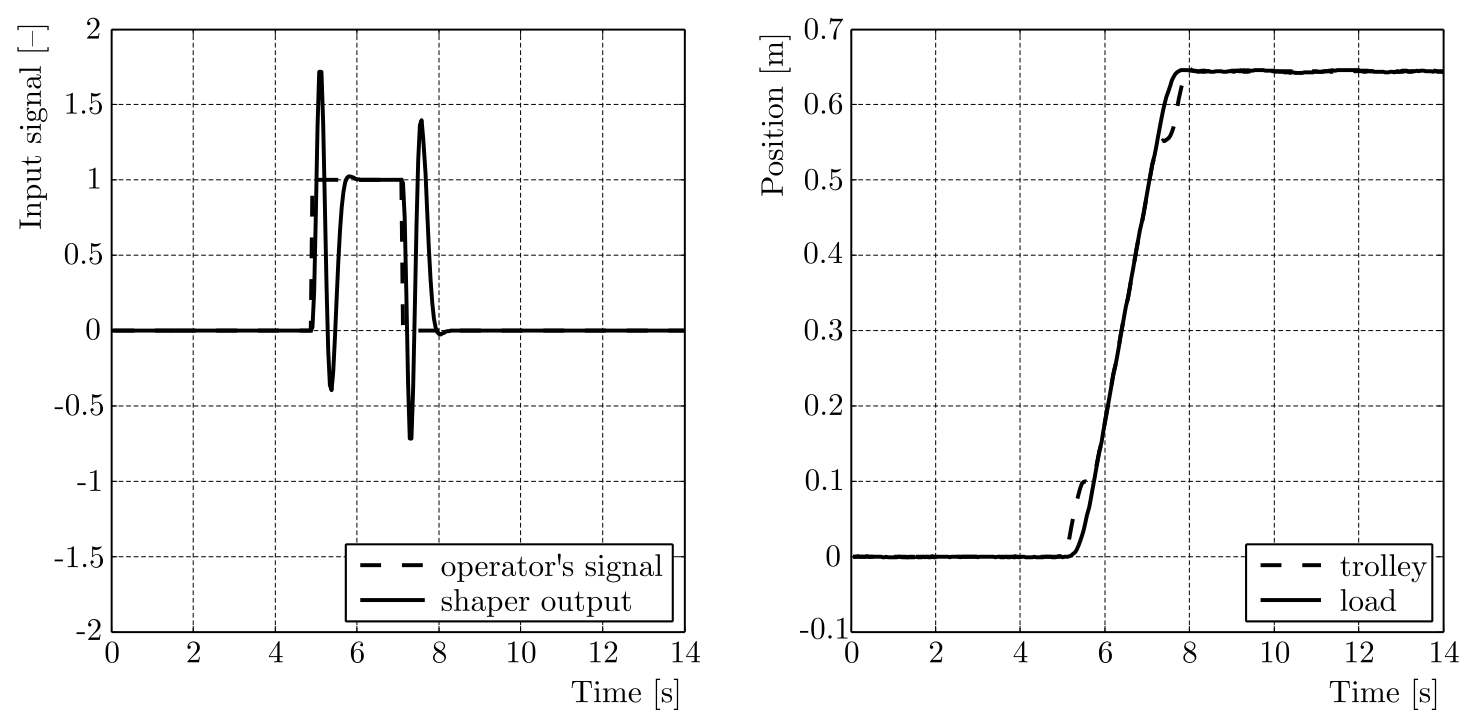

Fig. 12. Experiment the system response with the shaper

(a)

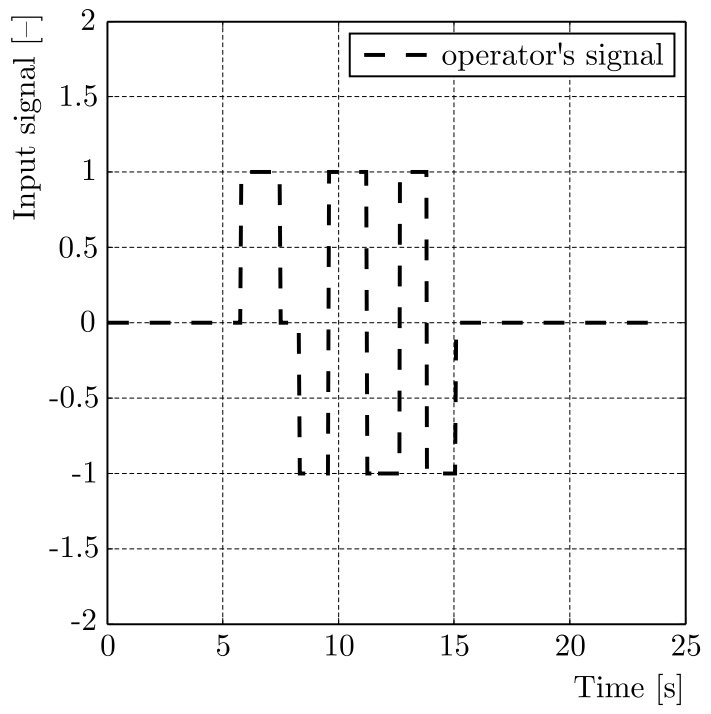

(b)

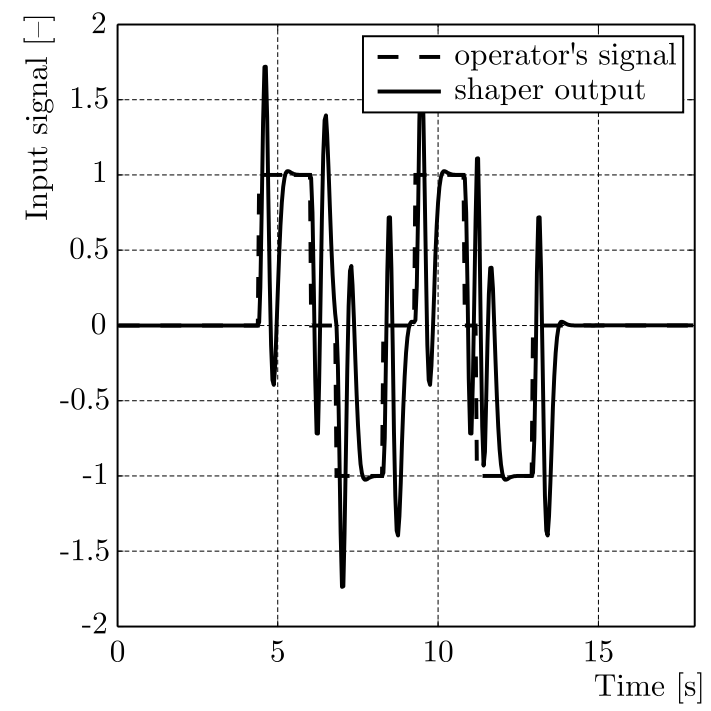

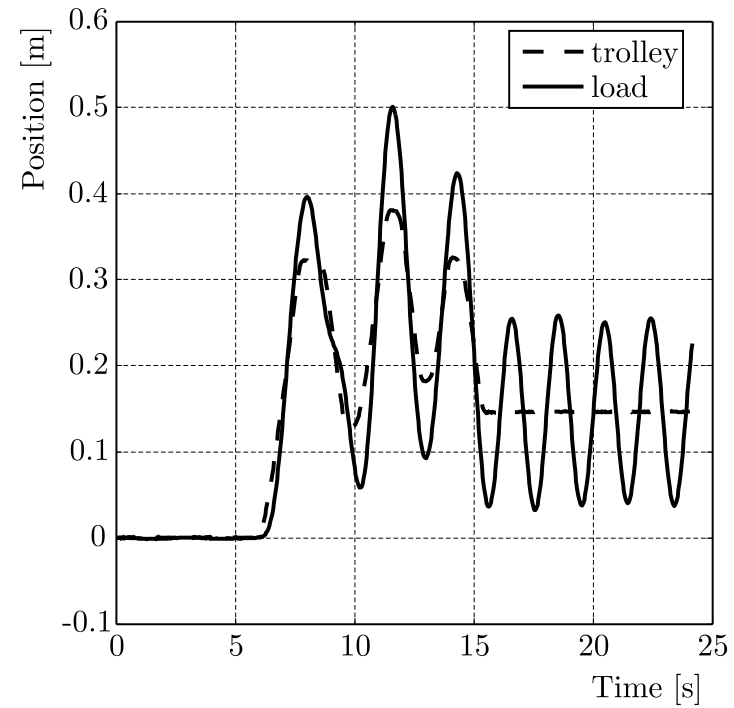

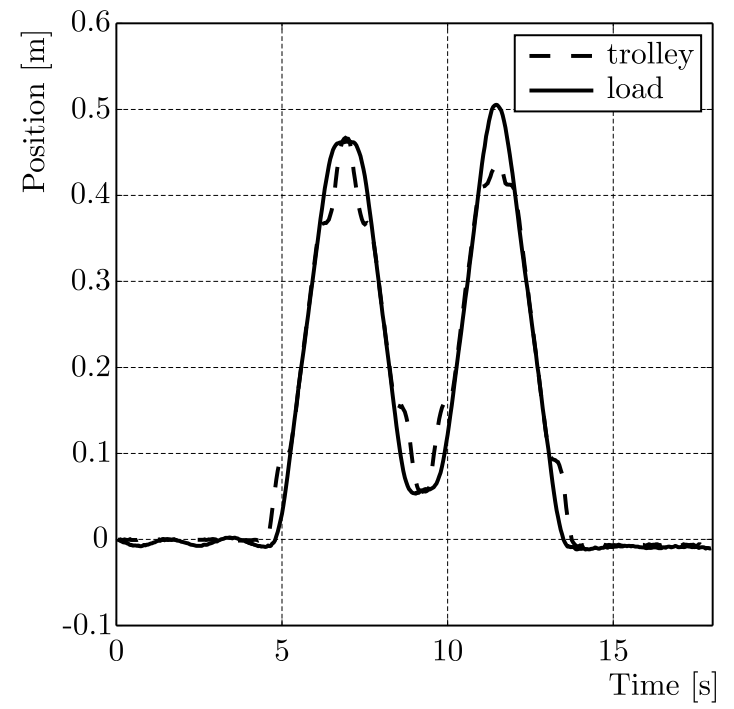

Fig. 13. Experiment a re-entry test (a) without the shaper, (b) with the shaper 


\section{Conclusions}

The presented approach to the design of input shapers combines the advantages of optimized precomputed control curves with the ability of on-line control and the re-entry property. It is capable of dealing with any optimization criteria or additional conditions. The length of produced shapers can be set arbitrarily only with respect to the limits of available power.

The synthesis of control input is based on the modified Laplace domain synthesis technique which takes into account the specific properties of chosen basis functions and their influence on the solution. Additional constraints and optimization criteria are dealt with using free weighting coefficients and/or parametric basis functions. The result of this part of the synthesis is an optimized precomputed curve. Using the finite time Laplace transform, this profile is transformed to the form of an on-line shaper. The shaper inherits the optimized properties of the precomputed curve and, in contrast to common shaping techniques, its length is not strictly determined as a multiple of the system natural frequency and can be set arbitrarily. It is important that the new result reduces the required power of the drive for the chosen positioning time. In its basic form, the resulting shapers represent velocity control, but they can be also easily modified to position, acceleration or jerk control. The theoretical results have been were verified by simulations and experiments.

Moreover, a detailed derivation of the necessary conditions for non-vibrational control (2.22) by Bhat and Miu (1991) has been was achieved. According to the best knowledge of the authors, it is published for the first time.

Acknowledgemment

The research has been was supported by the Czech Science Foundation, Project No. GAP101/11/2110 Advanced input shaping control for precise positioning of mechanisms.

\section{References}

1. Aspinwall D.M., 1980, Acceleration profiles for minimizing residual response, Journal of Dynamic Systems, Measurement and Control, 102, 3-6

2. Beneš P., 2012, Input Shaping Control with Generalised Conditions, Ph.D. Thesis (in Czech), Praha, ČVUT v Praze

3. BEneŠ P., VALÁŠEK M., 2008, Input shaping control with reentry commands on prescribed duration, Applied and Computational Mechanics, 2, 2, 227-234

4. Bнat S.P., Miu D.K., 1990, Precise point-to-point positioning control of flexible structures. Journal of Dynamics Systems, Measurements and Control, 4, 667-674

5. Bнат S.P., MiU D.K., 1991, Solutions to point-to-point control problems using laplace transform technique, Journal of Dynamics Systems, Measurement and Control, 113, 425-431

6. Chang P.H., Park H.S., 2005, Time-varying input shaping technique applied to vibration reduction of an industrial robot, Control Engineering Practise, 13

7. Heyden T., Woernle C., 2006, Dynamics and flatness-based control of kinematically undetermined cable suspension manipulator, Multibody System Dynamics, 16, 155-177

8. Kittnar Z., ValášEK M., 2004, Nonlinear control of crane manipulator, Proceedings of International Congress on Mechatronics, Prague: CTU, Faculty of Mechanical Engineering, 109-115

9. LAU M.A., PAO L.Y., 2003, Input shaping and time-optimal control of flexible structures, Automatica, 39, 893-900

10. Lewis F.L., 1992, Applied Optimal Control and Estimation, New Jersey, Prentice-Hall, Inc., ISBN 0-13-040361-X 
11. Miu D.K., 1993, Mechatronics, Electromechanics and Contromechanics, New York, Springer-Verlag

12. Neusser Z., VALÁšEK M., 2013, Control of the underactuated mechanical systems by harmonics, Proceedings of the ECCOMAS Thematic Conference on Multibody Dynamics 2013, Zagreb, University of Zagreb, 329-339

13. Osmic S., Berner M.O., Schwung A., Jost M., Monnigmann M., 2014, Flatness-based feedforward control for fast operating point transitions of compressor systems, Proceedings of 2014 IEEE Conference on Control Applications (CCA), 1753-1758

14. Park J.Y., Chang P.H., 2004, Vibration control of a telescopic handler using time delay control and commandless input shaping technique, Control Engineering Practise, 12

15. Piazzi A., Visioli A., 2002, Optimal dynamic-invesrion-based control of an overhead crane, IEE Proceedings Control Theory Applications, 149, 405-411

16. Post B.K., Mariuzza A., Book W.J., Singhose W., 2011, Flatness-based control of flexible motion systems, Proceedings of the ASME Dynamic Systems and Controls Conference, 843-850

17. Schindele D., Menn I., Aschemann H., 2009, Nonlinear optimal control of an overhead travelling crane, Proceedings of 18th IEEE Conference on Control Applications (CCA), 1045-1050

18. Singer N., SeERing W., 1990, Preshaping command inputs to reduce system vibration, Journal of Dynamics Systems, Measurements and Control, 112, 76-82

19. Singh T., Vadali S.R., 1993, Robust time-delay control, ASME Journal of Dynamic Systems, Measurement and Control, 115, 303-306

20. Singhose W.E., 1997, Command Generation for Flexible Systems, s.l.: Massachusetts Institute of Technology

21. Singhose W.E., Seering W., 1991, Generating vibration reducing inputs with vector diagrams, Proceedings of 8th IFToMM World Congress, 315-318

22. Sмith O.J.M., 1957, Posicast control of damped oscillatory systems, Poceedings of the IRE

23. Sugiyama S., Uchino K., 1986, Pulse driving method of piezoelectric motors, IEEE Journal, 637-640

24. VAlÁšEK M., 1995, Input shaping control of mechatronical systems, Proceedings of 9th World Congress IFTOMM, 3049-3052

25. Vyhlídal T., Kučera V., Hromčik M., 2012, Input shapers with uniformly distributed delays, Preprints of 10th IFAC Workshop on Time Delay Systems (accepted)

26. Wiederrich J.L., Roth B., 1974, Design of low vibration cam profiles, Conference on Cams and Cam Mechanisms, Liverpool

27. ZaVŘel J., VAlášEK M., 2004, Anti-sway rotational crane (derrick), Proceedings of International Congress on Mechatronics, Prague, CTU, Faculty of Mechanical Engineering, 101-108

28. Zimmert N., SAWodny O., 2010, Active damping control for bending oscillations of a forklift mast using flatness based techniques, Proceedings of 2010 American Control Conference, 1538-1543

Manuscript received April 2, 2015; accepted for print August 3, 2015 